\title{
L'exploitation de la multimodalité du message filmique dans le sous-titrage
}

\author{
Multimodality of filmic message in the subtitling
}

\author{
Paulina Borowczyk \\ Uniwersytet im. Adama Mickiewicza, Poznań \\ pborow@amu.edu.pl
}

\begin{abstract}
Audiovisual programs are meant to be seen and heard simultaneously. Screen translators have to take the verbal, acoustic and visual elements of audiovisual works into consideration. However they translate only the verbal content of the message. In the following article we focus on one of the main modalities for audiovisual translation, namely the subtitling. Our aim is to describe the specificity of screen translation and to think about the impact of subtitling process on the final product, that is on translation. We will show how translators take advantage of the presence of the verbal (dialogues) and the visual (screen) codes with the aim of reducing an original text.
\end{abstract}

Keywords: Mots-clés: screen translation, audiovisual translation, subtitling, technical limitations, filmic message

Aussi bien dans notre vie professionnelle que dans notre vie privée, nous sommes presque chaque jour devant un écran. «Les postes de télévision, le cinéma, les ordinateurs et les téléphones portables font maintenant partie intégrante de notre environnement social, fondé sur le pouvoir de l'écran. Nous les rencontrons à notre domicile, sur notre lieu de travail, dans les transports publics, les bibliothèques, les bars, les restaurants et les cinémas. Nous passons de nombreuses heures à regarder des écrans et à nous servir de programmes audiovisuels pour effectuer notre travail, développer et améliorer notre carrière professionnelle ou universitaire, nous divertir ou obtenir des informations » (Díaz Cintas, 2008 : 27). L'impact de l'image sur notre vie quotidienne est donc immense et nous ne pouvons plus nous en passer à l'heure actuelle. L'image est omniprésente, l'image nous entoure.

Et presque toujours l'image est accompagnée par la parole, au visuel est associé le verbal, que ce soit sous forme écrite ou orale. Nous sommes capables, en tant 
que spectateurs, de focaliser notre attention sur la couche visuelle et écouter simultanément les sons ; ou bien regarder un écran et lire en même temps les sous-titres ou d'autres inscriptions présentes à l'écran. Pourtant, " avec la parole est apparue le besoin de traduire » (Díaz Cintas, 2008 : 27). Afin de rendre compréhensibles et accessibles les programmes audiovisuels aux spectateurs provenant d'autres pays, il faut envisager un transfert linguistique. Cet article a pour but d'expliquer en quoi consiste la spécificité de la traduction pour les mass-médias et de présenter l'une des méthodes de transfert audiovisuel, notamment le sous-titrage et surtout nous aborderons une réflexion sur l'impact du sous-titrage sur le produit final, c'est-àdire la traduction. Au centre de notre étude seront les transformations que subit le texte d'arrivée lors du passage du code oral au code écrit d'une langue à l'autre. En proposant en premier lieu un survol sur les règles générales du sous-titrage, nous voudrions montrer, en nous appuyant sur les exemples provenant de différents films, comment les contraintes techniques de temps et d'espace influencentelles les décisions prises par un traducteur pour l'audiovisuel et comment le traducteur peut-il tirer profit de la présence de l'image, du savoir acquis par des spectateurs pendant la diffusion du film ou bien de la présence d'autres mots dans le contexte dans le but de raccourcir les sous-titres.

\section{SPÉCIFICITÉ DE LA TRADUCTION AUDIOVISUELLE}

Tout d'abord il faut mettre en valeur la complexité du message audiovisuel dans lequel plusieurs composantes sont présentes : le visuel, le verbal et le sonore. Tous ces éléments « peuvent avoir une grande importance au niveau de la signification du message » (Pettit, 2008 : 103). Il en résulte que, contrairement au traducteur des textes écrits, le traducteur audiovisuel ne peut pas traduire une émission audiovisuelle comme si c'était un texte écrit et il doit chercher «à rendre les énoncés de la bande son originale dans une autre langue en tenant compte du rapport entre les mots, l'image, la musique, les bruits, le tout véhiculé au moyen de la caméra et transmis à l'écran » (Pettit, 2008 : 103). Lors de la traduction des programmes audiovisuels, toutes ces composantes mentionnées peuvent, séparément ou collectivement, être des sources d'informations pour le traducteur.

Cette complexité du message médiatique qui passe par les différentes voies de communication concerne aussi le spectateur qui doit saisir le sens de façon rapide, d'un instant à l'autre tout en faisant attention aux données verbales, visuelles et sonores. Il ne peut pas, contrairement à un lecteur d'un livre ou d'un journal relire certains fragments du texte, revenir en arrière ou s'arrêter et réléchir sur telle ou telle autre formulation, un mot inconnu, etc.

En ce qui concerne la traduction d'un texte écrit, on va parler du passage d'un texte formulé en langue A vers un texte formulé en langue B tandis que dans le 
cas de la traduction audiovisuelle on va parler du transfert de tout un complexe sémiotique A que l'on traduit vers un complexe sémiotique B tout en sachant qu'une partie de ce complexe reste intacte (voir Tomaszkiewicz, 1999, 2000, 2006). Le message audiovisuel est considéré comme un tout inséparable et doit ainsi être traité dans le processus de la traduction. Il résulte de ceci que le traducteur audiovisuel ne peut pas se contenter de traduire uniquement les dialogues filmiques ou un commentaire sans regarder le film ou l'émission en question. Il traduit le verbal toujours dans un contexte audio et visuel. Pourquoi ? La plus importante des raisons est telle que le verbal entretient plusieurs relations avec d'autres signes non linguistiques qui l'accompagnent et le traducteur doit en être conscient. Ne pouvant pas les aborder toutes, nous pouvons donner comme exemple une émission à caractère éducatif et/ou informatif dans laquelle le commentaire verbal apporte des informations indispensables pour comprendre le visuel et en même temps l'image illustre les informations fournies par le verbal (pour plus d'informations sur la question des relations fondamentales entre le texte et la couche visuelle voir Tomaszkiewicz, 1999, 2006). Durant la diffusion de l'émission le verbal doit être synchronisé avec les informations découlant de la couche visuelle. Il en ressort que l'existence de ces différents rapports entre le mot et l'image peut se refléter dans la traduction même et, comme on le verra plus loin, peut aider le sous-titreur à amputer ou raccourcir certains segments du texte original.

En plus, à part le passage des sens entre le texte original et le texte d'arrivée, le traducteur audiovisuel est soumis aux contraintes techniques. On voit bien qu'il «se trouve devant plusieurs contraintes, imposées par la matière verbovisuelle, qui l'obligent d'envisager des opérations cognitives autres que le simple transfert du message, d'un système linguistique à un autre » (Tomaszkiewicz, 1999: 220). Les deux types de contraintes, notamment de temps et d'espace, liées à la nature même du programme audiovisuel auront une incidence immédiate sur le produit final, c'est-à-dire le texte d'arrivée. Dans la suite $\mathrm{du}$ présent article on verra de plus près quelles sont les opérations fondamentales $\mathrm{du}$ sous-titrage et de quelle manière le sous-titreur devra-t-il transformer les sous-titres par rapport aux dialogues originaux.

\section{LE SOUS-TITRAGE - DÉFINITION ET TRAITS CARACTÉRISTIQUES}

Commençons par une définition. Selon Díaz Cinats, «le sous-titrage interlinguistique peut être défini comme une pratique de traduction qui consiste à présenter, en général sur la partie inférieure de l'écran (...) un texte écrit qui s'attache à restituer : 
1) le dialogue original des locuteurs, qu'ils soient ou non à l'écran ;

2) les éléments discursifs qui apparaissent à l'image (les lettres, les insertions, les graffiti, les pancartes, les écrans d'ordinateurs et tout ce qui est du même ordre) ;

3) d'autres éléments discursifs qui font partie de la bande son, comme les chansons, les voix émanant de postes de télévision, de radios ou d'ordinateurs, etc. » (Díaz Cintas, 2008 : 28).

Comme nous l'avons déjà évoqué, dans les messages audiovisuels deux codes de communication principaux surgissent en même temps : l'image et le son. Cependant, une émission sous-titrée est un lieu de rencontre entre l'oral (le texte d'origine), l'image et les sous-titres. On oserait dire que le sous-titrage, aussi bien du point de vue d'un traducteur que d'un spectateur, est une opération particulièrement complexe. "L'interaction entre ces trois éléments, la capacité du (télé)spectateur à lire le texte écrit à une vitesse donnée ainsi que la taille de l'écran où sont diffusées les images déterminent les caractéristiques de base du support audiovisuel. Les sous-titres doivent impérativement être synchronisés avec l'image et le dialogue et doivent proposer une traduction conforme au dialogue de la langue source. Ils doivent également rester à l'écran suffisamment longtemps pour que les spectateurs aient le temps de les lire » (Díaz Cinats, 2008 : 28). Le sous-titrage est donc une forme d'adaptation cinématographique dans laquelle le traducteur doit respecter la concomitance de plusieurs codes à la fois (mots, image, musique, bruits) et tenir compte de leurs relations. Or, dans les programmes sous-titrés, «l'interaction entre les éléments cités est modifiée par la traduction inscrite au bas de l'écran. Gottlieb (1994: 106) souligne d'ailleurs que le succès du sous-titrage dépend du lien créé par rapport à l'image, tout comme la relation des dialogues avec l'image dans la version originale » (Pettit, 2008 : 103). C'est donc au traducteur de veiller à ne pas rompre le lien entre les sous-titres et les informations visibles sur l'écran.

En plus, le travail d'un sous-titreur est limité par deux contraintes majeures : le temps et l'espace. Ne pouvant pas présenter toutes les analyses détaillées des aspects formels des sous-titres nous présentons quelques règles générales qui devraient être respectées pendant l'opération du sous-titrage (à ce propos voir Gambier, 1996, 2001, 2003 ; Díaz Cintas, 1998, 2001, 2007 ; Lavaur et Şerban, 2008 ; Karamitroglou, 1998 ; Ivarsson, 1992, 2004). Pourtant, nous ne voulons en aucun cas néglier d'autres aspects importants comme la segmentation des sous-titres, la typographie détaillée (police des caractères, couleurs) ou encore le support utilisé (le cinéma, la télévision, l'Internet, le portable). Dans la suite nous nous limitons aux contraintes qui renvoient avant tout au temps de l'affichage des sous-titres à l'écran (ce qui est lié à la vitesse de lecture par le spectateur) et à leur longueur. Voici les principes qui doivent être pris en considération par un sous-titreur : 
A. Bien que les chiffres varient d'un pays à l'autre on peut admettre qu'en général, un sous-titre est constitué d'un maximum de deux lignes centrées au bas de l'écran.

B. Chaque ligne comporte entre 32 et 40 caractères (à savoir : les lettres, les signes et les espaces).

C. Un sous-titre peut être affiché pendant une seconde au minimum (une ligne) et pendant six secondes au maxiumum (deux lignes). En fait, cette contrainte est liée à une vitesse moyenne de lecture. «(...) il existe une relation directe entre la durée d'un sous-titre et le nombre de caractères qu'il peut contenir pour qu'il puisse être lu. Ces paramètres se basent sur une vitesse moyenne de lecture, nous ne pouvons pas lire la même quantité de texte si nous disposons de six secondes que si nous disposons de moins de temps. On estime que la vitesse de lecture actuelle est de trois mots par seconde. Donc, pour lire un sous-titre complet de deux lignes soit 70 caractères, il nous faudra au moins 4 secondes, puisqu'il contient environ douze mots. Si nous disposons de moins de temps, nous devrons envisager moins de caractères » (http://www.permondo.eu/fr/benevoles/ introduction-au-sous-titrage/). Et si un sous-titre est affiché plus de six secondes, le spectateur aura tendance à le relire.

D. « La ponctuation constitue un repère essentiel pour le spectateur. En effet, l'intonation de l'acteur (dans le cas de l'interrogation ou de l'exclamation) n'est pas toujours décelée par le spectateur qui ignore la langue. On veillera donc à un usage expressif (mais non abusif) du point d'interrogation et d'exclamation ainsi que du point de suspension » (http://lingalog. net/dokuwiki/_media/cours/sg/trad/methodest.pdf).

E. Il est conseillé de produire les phrases courtes et simples, «des segments organisés en unités syntaxiques les plus logiques possibles. Idéalement, un sous-titre devrait contenir une phrase complète, mais lorsque cela est impossible, la segmentation de chacune des lignes devrait être construite de manière à coïncider avec l'unité de sens la plus logique possible» (Orero, $2008: 63$ ). «Le texte d'arrivée ne devrait pas se transformer en télégramme en essayant de l'ajuster au nombre de caractères, il faut parvenir à une adaptation qui soit naturelle et correcte » (http://www.per mondo.eu/fr/benevoles/introduction-au-sous-titrage/).

En plus, le sous-titrage est la seule des méthodes de transfert audiovisuel qui « entraîne un changement dans le mode du discours qui passe de l'oral à lécrit » (Şerban, 2008: 28). On peut noter que "plusieurs différences peuvent être relevées entre l'utilisation du langage à l'écrit par comparaison avec l'oral. On peut par exemple remarquer qu'il y a normalement plus de redondance à l'oral, plus d'hésitation, de répétitions, de phrases inachevées, de nombreuses abrévia- 
tions ainsi qu'un registre moins soutenu » (Şerban, $2008: 92$ ), souvent familier et même vulgaire tandis que le langage écrit est beaucoup plus soigné, correct, où les principales règles de syntaxe sont respectées. Et on ne peut pas oublier que les sous-titres doivent former un tout cohérent avec d'autres éléments verbaux et non linguistiques et qu'ils ne seront pas lus par un spectateur « isolément, mais seront perçus en tant que composante du texte multimodal que constitue le programme audiovisuel » (Şerban, $2008: 92$ ).

Les facteurs mentionnés au dessus dont le traducteur doit tenir compte en préparant les sous-titres conduisent souvent à l'abrégement ou même à la suppression totale de certains éléments qui étaient présents dans le discours original. On estime que $30 \%$, même $40 \%$ du discours oral disparaît dans les sous-titres. Très souvent, à cause de la nécessité de condenser le texte, le spectateur connaissant la langue de départ, peut avoir l'impression que les soustitres ont été simplifiés et qu'à l'original les acteurs transmettent plus d'informations. Tomaszkiewicz propose de ramener, de façon générale, les techniques « résumatives » à deux opérations fondamentales :

- « la suppression totale de certains éléments, ayant une valeur secondaire dans la transmission du sens ;

- la transformation condensatoire qui permet de sauvegarder les contenus, mais de manière concise.

Ces deux opérations reposent sur la hiérarchisation de l'information, mais dans les mass-médias ce jugement du poids informatif dépend non seulement de l'information véhiculée linguistiquement mais aussi par d'autres moyens signifiants (...) » (Tomaszkiewicz, 1999 : 222).

C'est donc au sous-titreur de décider quelles informations sont indispensables pour comprendre le déroulement d'un film ou les relations entre les protagonistes et lesquelles il peut supprimer parce qu'elles sont de moindre importance, parce qu'elles sont répétées dans les dialogues originaux, parce qu'il en était question dans les scènes précédentes ou bien parce que les images, en tant qu'une source importante dans le film, aident le spectateur à comprendre certains aspects du film.

Dans la deuxième partie du présent article, étant donné que le travail d'un sous-titreur est soumis aux contraintes de temps et d'espace, nous proposons d'examiner de plus près les cas où le sous-titreur modifie la traduction en fonction 1) des signes visuelles, 2) du savoir acquis par les spectateurs durant la projection du film et 3) de la présence d'autres éléments verbaux pour omettre certains fragments du discours oral. Tous ces facteurs peuvent guider le traducteur dans ses choix linguistiques. Les exemples que nous citons ci-dessous proviennent aussi bien de l'ouvrage de Arkadiusz Belczyk Ttumaczenie filmów que des deux films, à savoir : Supercondriaque (pol. Przychodzi facet do lekarza 
qui est une comédie franco-belge réalisée par Dany Boon en 2014 et L'empire des loups (pol. Imperium wilków) qui est un film policier français de Chris Nahon sorti en 2005.

\section{INFLUENCE DE L'IMAGE SUR LES SOUS-TITRES}

«Comme les images ont la faculté de représenter les personnages et les endroits, ainsi que grâce au changement de scènes, l'écoulement temporel, les traducteurs vont traiter, en tant qu'information verbale secondaire, toute information qui se laisse déduire de l'écran » (Tomaszkiewicz, 1999: 223). Ainsi, les informations contenues dans les dialogues filmiques qui sont en plus visibles à l'écran vont disparaître dans les sous-titres :

Tableau 1

\begin{tabular}{|l|l|}
\hline \multicolumn{1}{|c|}{ Texte anglais (texte de départ) } & Texte polonais (texte d'arrivée) \\
\hline Am I safe in this crate ? & Jestem tu bezpieczna? \\
\hline
\end{tabular}

Dans le premier cas cité par Arkadiusz Belczyk dans son ouvrage Ttumaczenie filmów on voyait à l'écran une femme se cacher dans une caisse et on l'entendait demander : Am I safe in this crate ? Comme sa cachette était visible à l'écran, il n'était pas nécessaire d'en informer le spectateur polonais. Par conséquent, le sous-titreur a supprimé une expression nominale et l'a remplacée par un déictique simple tu (Belczyk, 2007: 29). Il a pu le faire grâce à la présence de l'image et il l'a fait sous l'influence de l'image.

Dans le deuxième extrait on a affaire à une scène à l'hôpital. L'un des docteurs aperçoit un nouveau patient sur le lit et demande à son collégue qui l'a amené à l'hôpital. En posant cette question il fait en même temps un geste pour indiquer le patient. Le geste utilisé par l'acteur souligne ce qu'il dit. Cet exemple montre, lui aussi, que le sous-titreur adapte son texte en fonction de ce qui se passe à l'écran :

Tableau 2

\begin{tabular}{|l|l|}
\hline \multicolumn{1}{|c|}{ Texte français (texte de départ) } & \multicolumn{1}{c|}{ Texte polonais (texte d'arrivée) } \\
\hline Qui a amené ici ce patient? & $\begin{array}{l}\text { Kto go przywiózt? } \\
\text { tiré du film : Supercondriaque }\end{array}$ \\
\hline
\end{tabular}


En plus, comme le traducteur sait que dans les scènes précédentes le spectateur a déjà vu le patient, il a décidé de remplacer le substantif par le pronom personnel go dans la version polonaise (Popek, 2015). L'image a donc complété la traduction.

Tableau 3

\begin{tabular}{|l|l|}
\hline \multicolumn{1}{|c|}{ Texte français (texte de départ) } & \multicolumn{1}{c|}{ Texte polonais (texte d'arrivée) } \\
\hline A :- Tiens. & B: Na co mi to? \\
B : - Pourquoi tu me donnes ça? & A: Na ból głowy. Ciagle masujesz skronie. \\
A : - T'as mal au crâne. Tu dois faire comme & tiré du film: Supercondriaque \\
ça depuis le matin. & \\
\hline
\end{tabular}

Dans le troisième exemple, le spectateur observe et entend le dialogue entre deux protagonistes : Romain (A) et son ami Marc (B). Ils travaillent tous les deux à l'hôpital. En s'occupant d'un patient, Romain aperçoit que Marc masse ses tempes. Il lui donne un médicament contre la douleur en disant Tiens (Popek, 2015). Cet énoncé n'a pas été traduit dans les sous-titres polonais parce que le geste est bien visible à l'écran et en plus, deux secondes plus tard le spectateur apprend de quel objet il s'agit.

D'après Tomaszkiewicz la liaison «entre les éléments du discours qui renvoient directement au cadre contextuel de l'énonciation et la situation même, qui dans les films est fournie par l'image, offre aux sous-titreurs des possibilités multiples, de compensation du verbal par le visuel » (Tomaszkiewicz, 1993 : 190). Les trois exemples cités prouvent qu'afin de pouvoir supprimer certains éléments présents dans le discours oral, le sous-titreur devait s'assurer que les sous-titres correspondent à ce qui se passe à l'écran et que le récepteur du film sous-titré comprendrait la scène en question.

\section{INFLUENCE DU SAVOIR DES SPECTATEURS SUR LES SOUS-TITRES}

En principe, il s'agit des situations dans lesquelles le sous-titreur peut supprimer certains éléments présents dans le discours oral grâce au savoir que les spectateurs acquièrent, peu à peu, pendant la projection du film. En supposant que le spectateur suive l'action du film (aussi bien sa couche visuelle que verbale) de manière attentive, il est ensuite capable d'activer les informations nécessaires à un moment convenable pour comprendre le contexte de la situation 
en question et de reconstruire les éléments supprimés dans les sous-titres. Voici les exemples illustrant ce phénomène :

Tableau 4

\begin{tabular}{|l|l|}
\hline \multicolumn{1}{|c|}{ Texte français (texte de départ) } & \multicolumn{1}{|c|}{ Texte polonais (texte d'arrivée) } \\
\hline $\begin{array}{l}\text { Vous avez des relations sexuelles normales avec } \\
\text { votre mari ? }\end{array}$ & $\begin{array}{l}\text { Czy utrzymujecie normalne stosunki seksualne? } \\
\text { tiré du film : L'empire des loups }\end{array}$ \\
\hline
\end{tabular}

Cet extrait a lieu lorsque Anna, l'héroïne principale du film est interrogée par une psychologue à propos des relations avec son mari. Pour le spectateur qui observe dès le début les relations et les interactions entre les protagonistes il est évident qu'il s'agit du mari d'Anna (Nowak, 2006). L'omission de ce substantif dans les sous-titres polonais ne perturbe, en aucun cas, la bonne compréhension de la question.

Dans l'exemple suivant, la signification est aussi évidente pour le spectateur par rapport aux scènes qui précèdent.

Tableau 5

\begin{tabular}{|l|l|}
\hline \multicolumn{1}{|c|}{ Texte français (texte de départ) } & \multicolumn{1}{|c|}{ Texte polonais (texte d'arrivée) } \\
\hline $\begin{array}{l}\text { Tu voudrais qu'il te pardonne alors que tu lui } \\
\text { as volé la drogue? }\end{array}$ & $\begin{array}{l}\text { Myślisz, że daruje ci kradzież? } \\
\text { tiré du film : L'empire des loups }\end{array}$ \\
\hline
\end{tabular}

Dans le cinquième extrait le sous-titreur a décidé de supprimer le substantif la drogue dans les sous-titres polonais. En fait, les spectateurs connaissent déjà l'histoire du vol qui a été montrée dans les scènes précédentes. Ils savent qu'il est question du vol de la drogue et non pas de quelque chose d'autre (Nowak, 2006). Le sous-titreur a jugé cette information comme redondante par rapport au savoir des spectateurs.

Tableau 6

\begin{tabular}{|l|l|}
\hline \multicolumn{1}{|c|}{ Texte français (texte de départ) } & \multicolumn{1}{c|}{ Texte polonais (texte d'arrivée) } \\
\hline $\begin{array}{l}\text { Je vais au commissariat où elle est amenée. J'y y yadę na komisariat. Sam! } \\
\text { vais seul. }\end{array}$ & $\begin{array}{l}\text { Jare du film : L'empire des loups } \\
\text { tiré }\end{array}$ \\
\hline
\end{tabular}


Dans le cas ci-dessus on observe la dispariton de la proposition subordonnée circonstancielle de lieu dans les dialogues sous-titrés. Elle a été jugée inutile parce qu'elle était redondante par rapport au contexte de l'histoire. Les spectateurs savent déjà que Anna, la principale protagoniste dans le film a été amenée au commissariat (Nowak, 2006).

Dans ce qui suit nous pensons démontrer comment d'autres éléments verbaux présents dans les dialogues originaux permettent de prévoir une version raccourcie de la traduction.

\section{INFLUENCE D'AUTRES ÉLÉMENTS VERBAUX SUR LES SOUS-TITRES}

Les répétitions constituent un des traits caractéristqiues du discours oral. L'échange des paroles et toute sorte d'interaction verbale se caractérisent par « des opérations telles que répétitions, reprises, corrections, reformulations autoou hétéro-initiées, pour assurer une bonne intercompréhension entre les interlocuteurs » (Tomaszkiewicz, 1999: 226). Les sous-titres, par contre, doivent être condensés. C'est pour cela « tous les éléments qui sont repris, corrigés, répétés ou reformulés, n'apparaissent pas dans les sous-titres qu'une seule fois » (1999 : 226). La suppression de différents types de répétitions est très fréquent dans la traduction filmique :

Tableau 7

\begin{tabular}{|l|l|}
\hline \multicolumn{1}{|c|}{ Texte français (texte de départ) } & \multicolumn{1}{c|}{ Texte polonais (texte d'arrivée) } \\
\hline $\begin{array}{l}\text { A : Ils l'ont torturé pendant des heures. } \\
B: \text { Comment ça des heures } ?\end{array}$ & $\begin{array}{l}\text {-Torturowali ja godzinami. } \\
\text { - Jak to? } \\
\text { tiré du film : L'empire des loups }\end{array}$ \\
\hline
\end{tabular}

Tableau 8

\begin{tabular}{|l|l|}
\hline \multicolumn{1}{|c|}{ Texte français (texte de départ) } & \multicolumn{1}{c|}{ Texte polonais (texte d'arrivée) } \\
\hline A : Quel genre de recherches? & - Jakie badania? \\
$B: \underline{\text { Des recherches de secrète. }}$ & $\begin{array}{l}\text { - Tajne. } \\
\text { tiré du film }: \text { L'empire des loups }\end{array}$ \\
\hline
\end{tabular}

Dans les extraits ci-dessus, il s'agit de la suppression d'une partie de l'énoncé qui a été déjà utilisé dans la phrase précédente (Nowak, 2006). 
L'omission des ces éléments dans le but de produire un effet de raccourcissement n'entraîne, d'aucune manière, l'incompréhension du texte d'arrivée.

En plus, il est important de souligner que les répétitions « jouent avant tout un rôle expressif pour manifester l'excitation, la colère, la peur, l'énervement, la surprise de l'énonciateur » (Tomaszkiewicz, 1993 : 52). Dans les deux cas suivants les répétitions qui aparaissent dans les dialogues originaux expriment successivement l'énervement (tableau 9) ou le mépris et la peur envers la personne évoquée (tableau 10). Les émotions qui se réflètent dans les paroles des protagonistes, sont aussi bien visibles sur leurs visages. Grâce à l'image, les répétitions peuvent être omises :

Tableau 9

\begin{tabular}{|l|l|}
\hline \multicolumn{1}{|c|}{ Texte français (texte de départ) } & \multicolumn{1}{|c|}{ Texte polonais (texte d'arrivée) } \\
\hline $\begin{array}{l}\text { Monsieur, vous m'entendez? Monsieur, si vous } \\
\text { m'entendez, serrez la main! Monsieur! }\end{array}$ & $\begin{array}{l}\text { Styszy mnie pan? Jeśli tak, prosze ścisnaćc mi } \\
\text { dtoń. } \\
\text { tiré du film : Supercondriaque }\end{array}$ \\
\hline
\end{tabular}

Tableau 10

\begin{tabular}{|l|l|}
\hline \multicolumn{1}{|c|}{ Texte français (texte de départ) } & \multicolumn{1}{|c|}{ Texte polonais (texte d'arrivée) } \\
\hline $\begin{array}{l}\text { C'est un tueur. Un tueur plus cruel que tu peux } \\
\text { imaginer. }\end{array}$ & $\begin{array}{l}\text { Morderca zdolny do nieludzkiego okrucieństwa. } \\
\text { tiré du film : L'empire des loups }\end{array}$ \\
\hline
\end{tabular}

Les répétitions, « comme elles relèvent de l'expressivité, elles peuvent être compensées par les données non verbales, provenant de l'écran, d'où (...) leur faculté de disparition presque totale dans les sous-titres » (Tomaszkiewicz, 1993: 52). Dans le septième exemple un practicien hospitalier essaie de maintenir le contact avec un patient qui vient d'être transporté à l'hôpital. Il est très engagé et manifeste sa nervosité. Le sous-titreur a donc supprimé le titre monsieur et la proposition conditionnelle en faisant référence à la question qui la précède (Popek, 2015). Par contre, dans le dernier cas, la répétition du mot un tueur accompagnée par la mimique de l'actrice accentue le sentiment de peur et de mépris envers la personne dont parle la protagoniste. Elle sert à souligner l'importance de l'énoncé (Nowak, 2006). Le traducteur a pu procéder aux amputations textuelles "grâce à l'ancrage de ces dialogues dans la situation énonciative, constamment visualisée à l'écran » (Tomaszkiewicz, 1999 : 229). 


\section{CONCLUSION}

Comme le remarque Gambier, «trois problèmes fondamentaux se posent dans les transferts linguistiques audiovisuels, à savoir la relation entre images, sons et paroles dans un texte multimodal, la relation entre langue(s) étrangère(s) et langue d'arrivé (donc, la traduction), ainsi que la relation entre code oral et code écrit, qui exige une évaluation des normes de l'écrit dans des situations où les messages sont éphémères (par exemple, les sous-titres) et où ils sont présentés en parallèle avec le déroulement d'une interaction orale que l'on voit dans les images et que l'on peut entendre dans la langue d'origine » (Şerban, 2008: 88). Les exemples qu'on a choisi démontrent l'impact des contraintes techniques sur la traduction en forme des sous-titres. Étant obligé de condenser le texte d'arrivée, le sous-titreur est obligé d'abréger le texte d'arrivée. Pourtant, le sens dans le message médiatique est véhiculé non seulement à travers les éléments verbaux mais aussi non-verbaux, comme l'image et et le son. On a voulu démontrer que ne pouvant pas traduire tout le discours oral, le soustitreur profite de la présence d'autres signes non linguistiques (avant tout les données visuelles), des facteurs extérieurs au film (savoir des spectateurs acquis pendant la diffusion du film) et d'autres éléments verbaux. Par conséquent, il peut raccourcir le dialogue original afin de laisser le temps au spectateur de lire les sous-titres. 


\section{BIBLIOGRAPHIE}

Aubert, J.-P., Marti M., de Nice, U. Quelques conseils pour le sous-titrage. http://lingalog.net/ dokuwiki/_media/cours/sg/trad/methodest.pdf

Belczyk, A. (2007). Ttumaczenie filmów. Wilkowice : Wydawnictwo Dla Szkoły.

Diaz Cintas, J. (2008). Pour une classification des sous-titres à l'époque du numérique. In J.-M. Lavaur \& A. Şerban (Eds.), La traduction audiovisuelle. Approche interdisciplinaire du sous-titrage (pp. 27-41). Bruxelles : De Boeck.

Introduction au sous-titrage. http://www.permondo.eu/fr/benevoles/introduction-au-sous-titrage/

Nowak, A. (2006). Les éléments des dialogues filmiques qui subissent la suppression pendant la traduction en forme des sous-titres sur l'exemple du film français "L'empire des loups » (praca licencjacka napisana pod kierunkiem dr Pauliny Borowczyk w Zakładzie Traduktologii i Badań nad Kanadą Frankofońską). Poznań.

Orero, P. (2008). Le format des sous-titres : les mille et une possibilités. In J.-M. Lavaur \& A. Şerban (Eds.), La traduction audiovisuelle. Approche interdisciplinaire du sous-titrage (pp. 55-67). Bruxelles : De Boeck.

Pettit, Z. (2008). Le sous-titrage : le rôle de l'image dans la traduction d'un texte multimodal. In J.-M. Lavaur \& A. Şerban (Eds.), La traduction audiovisuelle. Approche interdisciplinaire du sous-titrage (pp. 101-111). Bruxelles : De Boeck.

Popek, A. (2015). Analyse des opérations de suppression et de condensation pendant le processus de sous-titrage (praca licencjacka napisana pod kierunkiem dr Pauliny Borowczyk w Zakładzie Traduktologii i Badań nad Kanadą Frankofońską). Poznań.

Şerban, A. (2008). Les aspects linguistiques du sous-titrage. In J.-M. Lavaur \& A. Şerban (Eds.), La traduction audiovisuelle. Approche interdisciplinaire du sous-titrage ( $\mathrm{pp}$. 85-99). Bruxelles : De Boeck.

Tomaszkiewicz, T. (2000). Le sens et l'information à transmettre dans la traduction des messages verbo-visuels. Studia Romanica Posnaniensia, XXV/XXVI, 305-315.

Tomaszkiewicz, T. (1999). Texte et image dans les communications aux masses. Poznań: Wydawnictwo Naukowe UAM.

Tomaszkiewicz, T. (1993). Les opérations linguistiques qui sous-tendent le processus de soustitrage des films. Poznań : Wydawnictwo Naukowe UAM. 
